\title{
Multi-Robot Systems Design and Implementation Based On Psoc5
}

\author{
Jiansheng Peng ${ }^{*}$ \\ Department of Physics and Mechanical \& Electronic Engineering, Hechi University, Yizhou 546300, China
}

\begin{abstract}
The direction for the robot toward positioning and navigation, multi-sensor, human-computer interaction, the overall control, network robots, multi-robot and the rapid developing of intelligent technology to improve the limitations of a single robot, designed with intelligent, low power consumption and multi-overall multi-robot control system sensors. Wireless sensor networks, multi-robot technology, multi-sensor technology, human-computer interaction techniques and overall control system on chip technology to CC2530 and PSoC5 for the master core, temperature, humidity, ultrasonic, ambient light and infrared proximity detection, camera and electronic compass and other sensors in real-time environment in which the robot to collect temperature, humidity, distance, light intensity, image, orientation and other information. After commissioning and field testing the entire system to use, the system is stable, human-computer interaction convenient, reliable communication and information gathering to diversify, to achieve the desired design features and has achieved good results, can be used for civil and industrial intelligent surveillance applications.
\end{abstract}

Keywords: Multi-robot systems, PSoC5, CC2530, Multi-sensor technology.

\section{INTRODUCTION}

Driven by the rapid development of wireless sensor network technology, short-range wireless communications technology, multi-sensor technology, embedded technology, system-on-chip technology and microelectronics technology [1-4], a lot of technical and academic integration, application of robotic systems in the current study and design of multirobot systems has become one of the hottest areas of research at home and abroad [5-7]. Sources of information for the previous single robot system, unstable operation and control of inconvenient limitations [3,5], driven by domestic and foreign markets, so that the robot along the navigation, multi-sensor, a service robot, the overall control, network robot and direction of development of multi-robot $[2,4,5$, 8], so that multi-robot systems with intelligent, low cost, low power consumption and high reliability [3]. Especially designed Programmable System on Chip PSoC5 and $2.4 \mathrm{GHz}$ IEEE802.15.4 / RF4CE / ZigBee CC2530 second-generation chip-based multi-robot system combines wireless sensor networks, multi-robot technology, multi-sensor technology, human-computer interaction overall control technology and system-on-chip technology $[1-5,8]$, particularly conducive to the robot to detect complex or unknown environments, overall control, positioning, and human-computer interaction.

\section{OVERALL SYSTEM DESIGN}

The main objective of the multi-robot system design is to achieve human-computer interaction overall control multiple robots gathering information on various types of sensors, as well as PC and multi between robots. The system uses PSoC5, CC2530, multi-robot and multi-sensor technology for the design, the system is determined on the basis of wireless communications based on the design of the corresponding coordinator, routers, and terminal equipment modules. Wherein, CC2530 ZigBee wireless communication network, a network message is a short delay, a high data transmission reliability and robustness of a network of high mesh network topology as showed in Fig. (1).

\subsection{Coordination Module}

Coordination of multi-robot system module is the first and unique network equipment, is responsible for starting the entire network, configure the network member addresses, network maintenance and other functions [9] In addition, it is also responsible for data communication with the $\mathrm{PC}$ via UART manage all robots, human-computer interaction to achieve overall control. Among them, the system coordinator module block diagram showed in Fig. (2), which consists of CC2530, PC machine, Nokia5110 LCD module and 2.4G antennae.

\subsection{Router Module}

Router module task of multi-robot system is relatively heavy, which has extended network, allowing other devices to adhere to the network, multi-hop routing and assisting battery-powered terminal device communications and other functions [10]. Which is responsible for CC2530 via SPI interface PSoC5 data communications, control and management trolley movement, multi-sensor signal acquisition and processing and other tasks, PSoC5 realize ultrasonic module, camera module, QVGA TFT modules, modules and components to control and manage. Router module of the system 


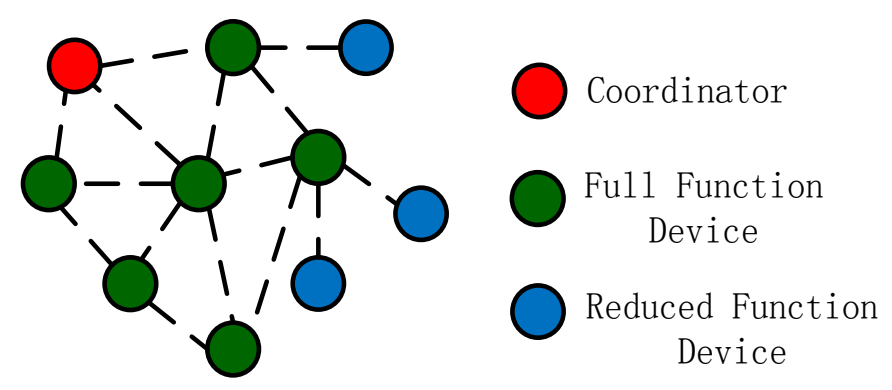

Fig. (1). Mesh network topology diagram.

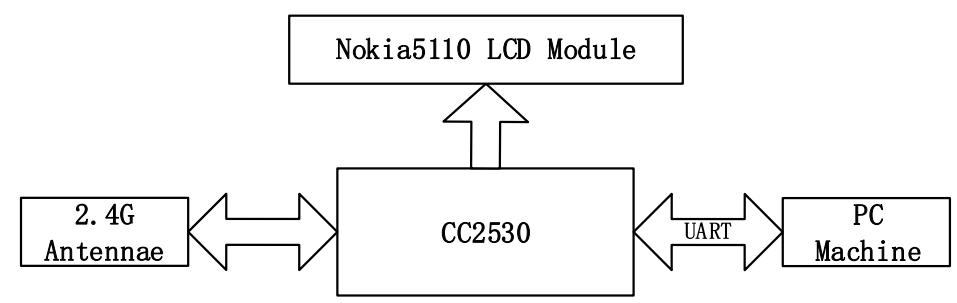

Fig. (2). System block diagrams of the module coordinator.

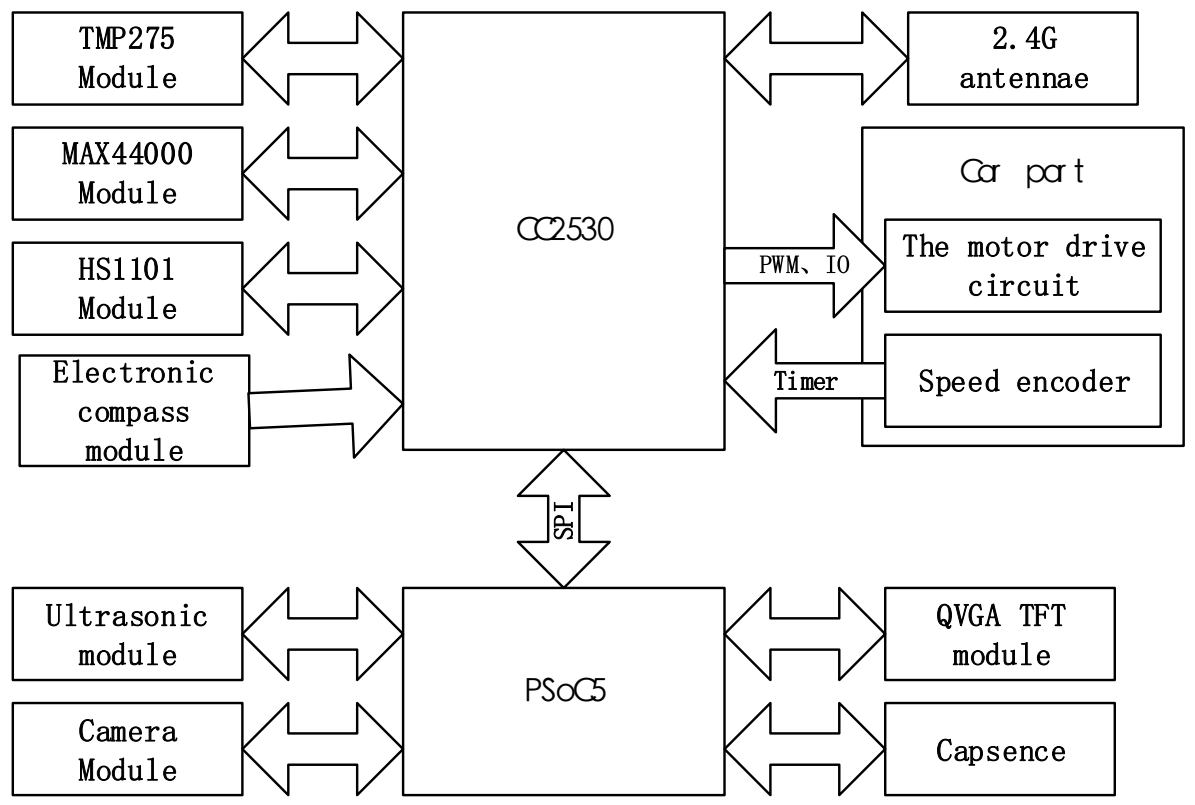

Fig. (3). Module system blocks diagram router.

by CC2530, PSoC5,2.4G antenna, car part, QVGA TFT display module, and various sensors modules, the system block diagram shown in Fig. (3).

\subsection{Terminal Equipment Module}

Terminal equipment of multi-robot system, there is no specific structure to maintain the function of the network, and therefore, the structure and function of the terminal device are relatively simple, the main signal acquisition and processing of controlling the robot, and some sensors. Its router module less than some features, mainly by CC2530, 2.4G PCB antenna, car parts, steering module, lithium battery module and a number of sensor modules, specific sys- tem block diagram presented in Fig. (4). In addition, terminal equipment modules can add additional sensor modules depending on the actual situation, but also to enter a low-power standby mode.

\section{SYSTEM HARDWARE DESIGN}

\subsection{Master Chip Hardware Designs}

\subsubsection{CC2530 Core Circuit Design}

The multi-robot system uses the TI's latest secondgeneration SoC chip CC2530, compared with other chip, 


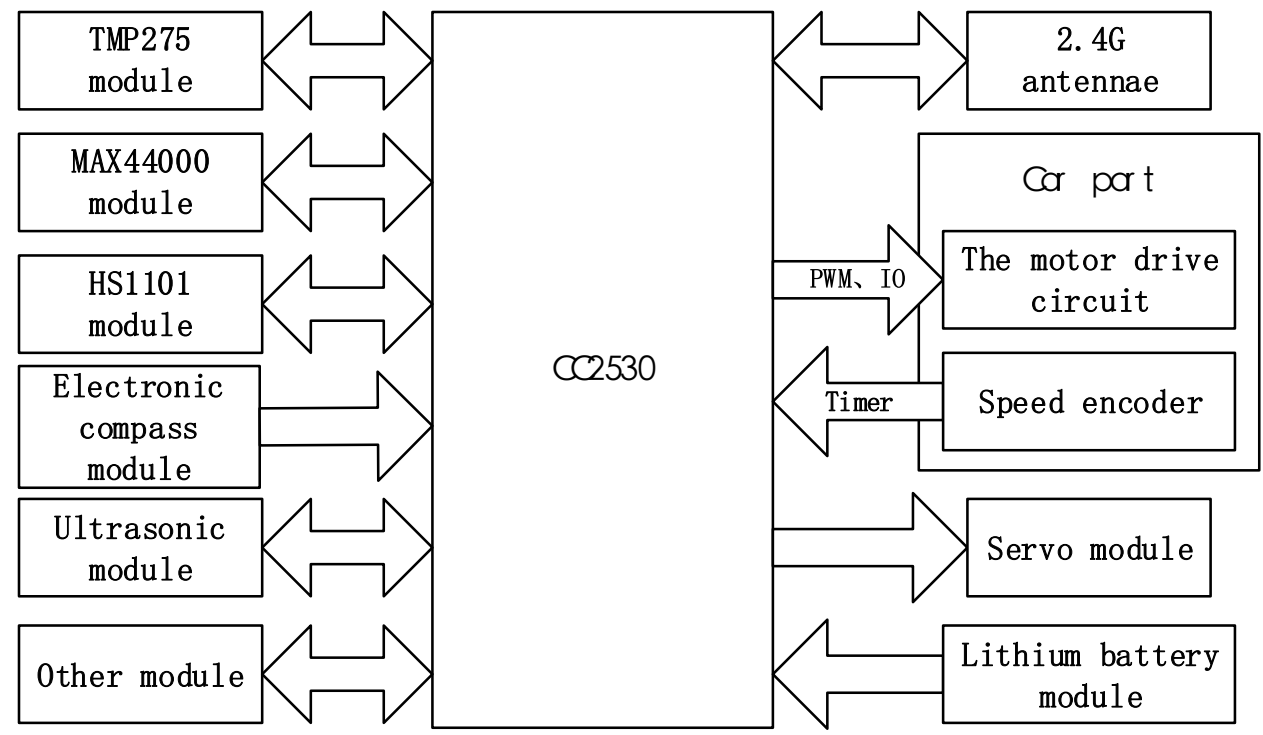

Fig. (4). Terminal equipment module system block diagram.

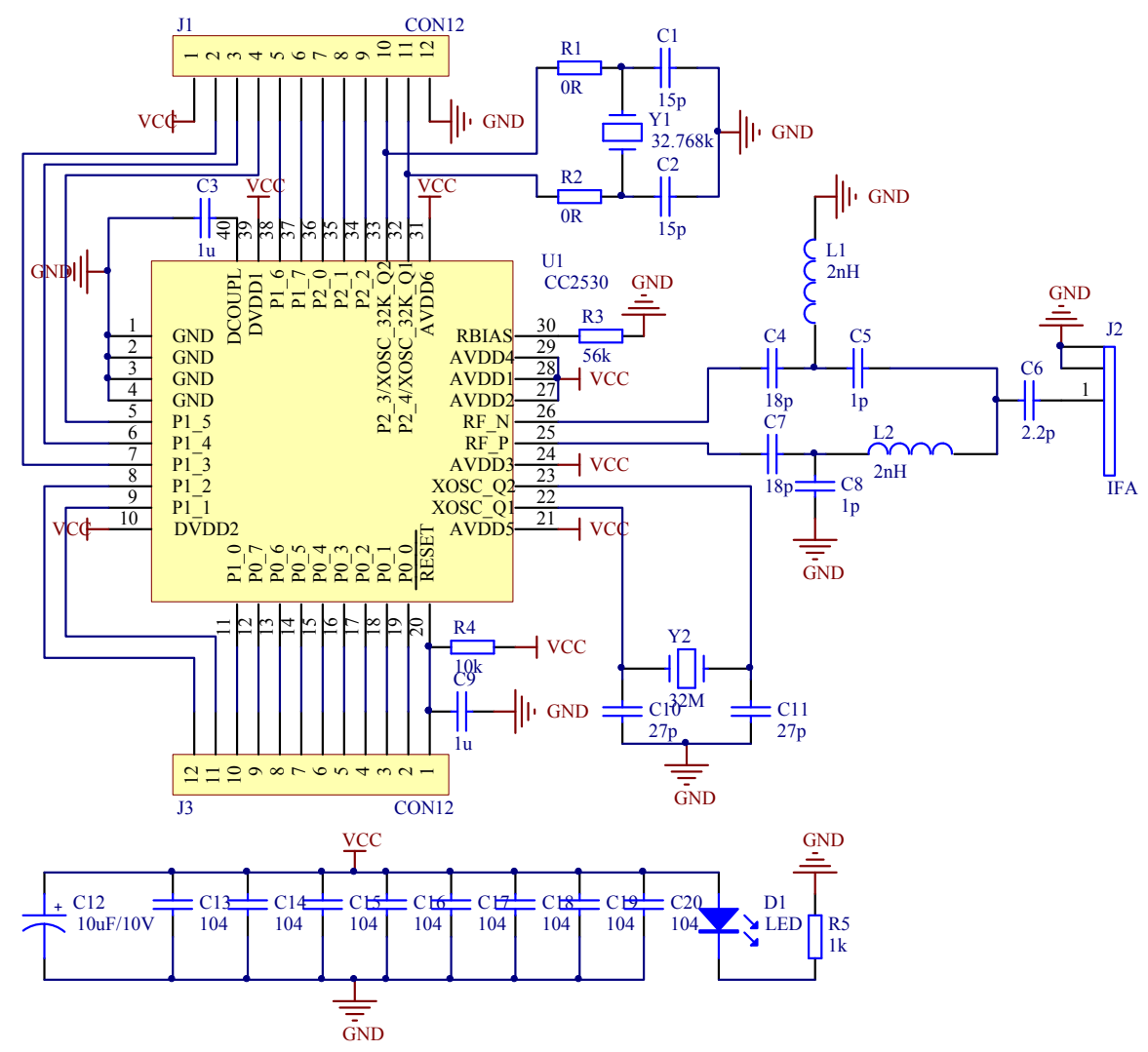

Fig. (5). CC2530 core diagram.

CC2530 has the support of low-power wireless communication, has a strong RF performance, programmable up to $256 \mathrm{~KB}$ Flash, up to $101 \mathrm{~dB}$ link quality and support for multiple network protocol stack (eg Simplici TI, Z-Stack or Remo TI) $[11,12]$. In this design, CC2530 chip peripheral interface control in addition to using various types of sensors, the most important feature is its wireless communications technology, the core of the circuit shown in Fig. (5).

\subsubsection{PSoC5 Hardware Design and Configuration}

The multi-robot systems using the US company's Cypress PSoC Cypress most powerful, the most resource-rich devices CY8C55 series chips, which have a superior resource integration [13]. Use CY8C55 series chip design, both to avoid the complicated wiring, and reduce the use of external components, reducing design costs and improve the reliability 


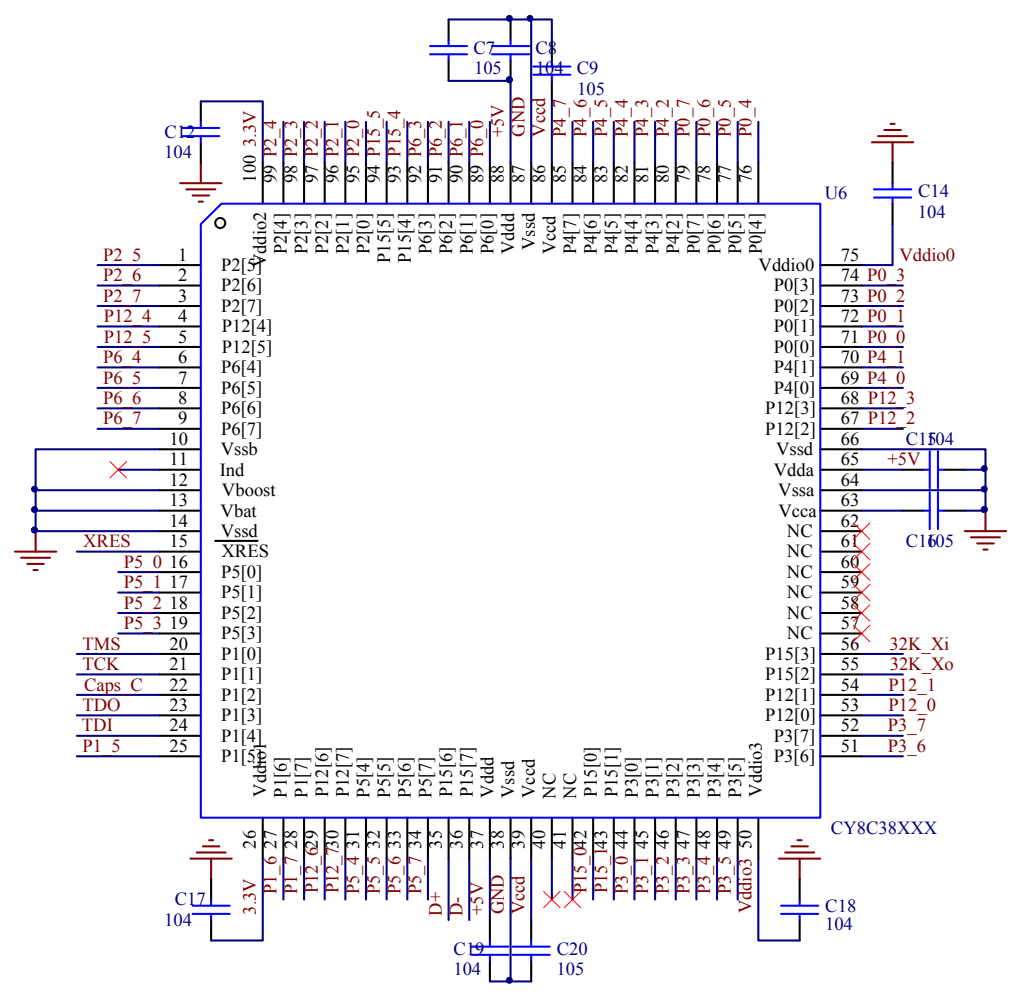

Fig. (6). PSoC5 core diagram.
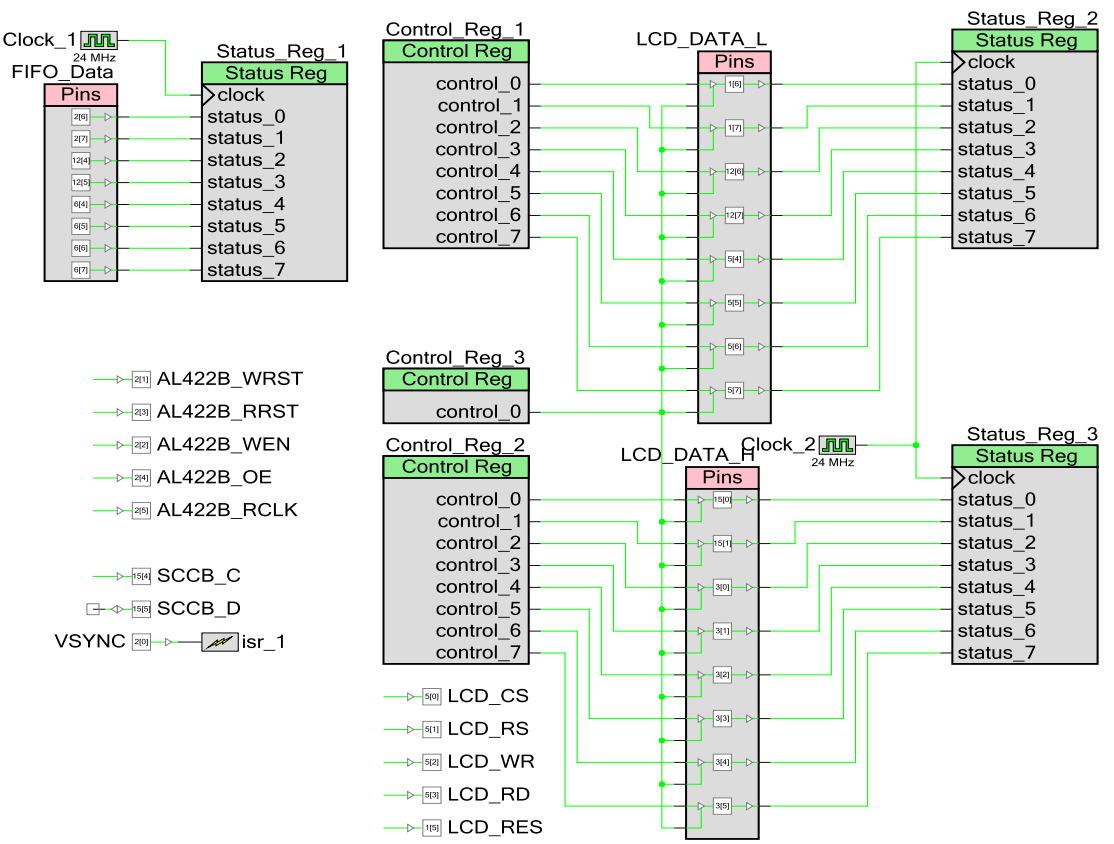

Fig. (7). PSoC5 resource allocation figure.

and stability of the system is very suitable for multi-robot system design. Wherein, PSoC5 core circuit shown in Fig. (6).

In addition, Cypress has provided the latest hierarchical schematic design entry tool PSoC Creator 2.0, which contains a number of basic components and features. Designers can very easily create their own system-level design [12]. The system of internal hardware resources PSoC5 configured, including three state registers Status_Reg, I communicate with two control registers Control_Reg and with multiple functional modules / $\mathrm{O}$ pins, the exact configuration shown in Fig. (7). 


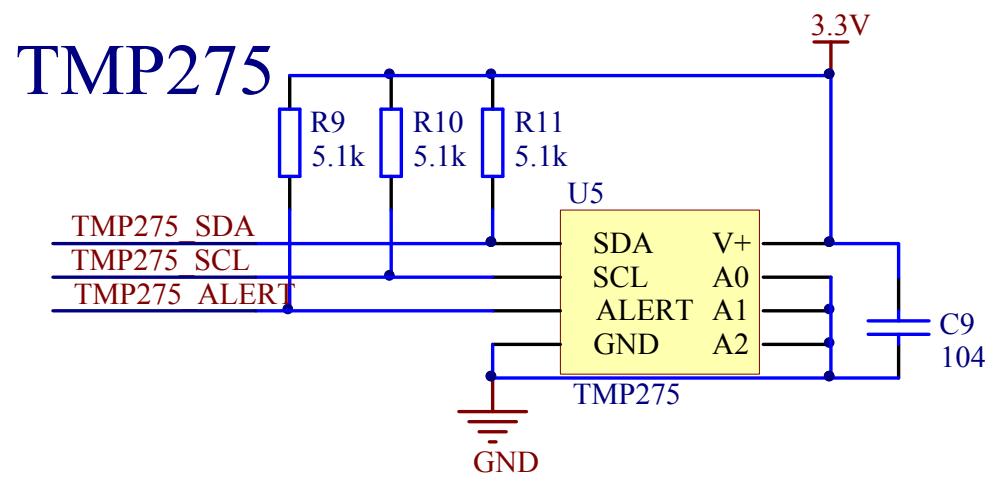

Fig. (8). TMP275 temperature measurement circuit schematics.

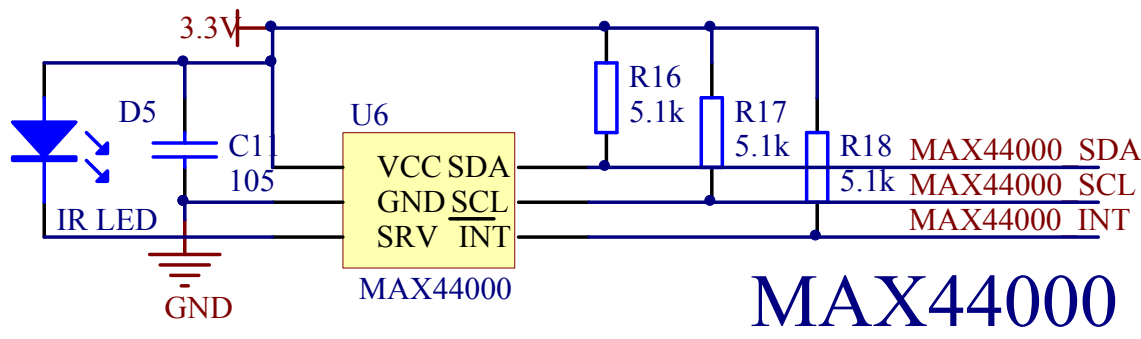

Fig. (9). MAX44000 ambient light and infrared proximity detection circuit diagram.

\subsection{Sensor Module Hardware Design}

\subsubsection{Temperature Sensor}

Temperature detection part of multi-robot system using TI's high-precision, high stability, low power consumption, resolution up to $0.0625{ }^{\circ} \mathrm{C}$, temperature range $-40{ }^{\circ} \mathrm{C} \sim+125$ ${ }^{\circ} \mathrm{C}$, the maximum temperature error of only $\pm 0.5{ }^{\circ} \mathrm{C}$ new temperature sensor TMP275, its temperature measurement circuit diagram shown in Fig. (8).

\subsubsection{Ambient Light and Infrared Proximity Sensor}

The system uses Maxim Integrated Products (Maxim Integrated Products) launched capable of measuring 0.03 Lux $\sim 65535$ Lux range of ambient light intensity and infrared proximity sensor MAX44000. Analog human eye to identify ambient light and infrared proximity detection. The circuit shown in Fig. (9).

\subsubsection{Other Sensor Module}

In addition, the system also uses employ 555 Drive HS1101 [14] CMOS process humidity sensor, measuring range from 0.02 to $5.50 \mathrm{~m}$, the measurement accuracy of up to $1 \mathrm{~cm}$ of the DYP-ME007 V1 ultrasonic ranging module, the OV7725, AL422B and lens configuration camera module, Honeywell's measurement range of -8 Gauss $\sim+$ measurements of the geomagnetic field of the electronic compass module 8 Gauss's HMC5883L composition.

\subsection{Robot Parts of the Hardware Design}

\subsubsection{The Motor Drive Circuit Design}

Robot chassis of motor used in this system is the high torque motor 260 . In order to make the robot more flexible, mobile and more robust performance, the design will ipsilateral motor driven in parallel, and containing four BTS7960 dual H-bridge motor Drive circuit drives in parallel, using dual PWM controlled, specific circuit shown in Fig. (10) are shown.

\subsubsection{Encoding Speed Circuit Design}

Multi-robot systems used crawler robot chassis of each gear speed encoder will output two speed difference of $90^{\circ}$ and with the same frequency square-wave changes. The signal output of the encoder gear speed acquisition and analysis, to understand the state of motion of the robot, and the motor drive circuit so as to form a closed loop system, and further is stable, easy to control the robot.

The system for the collection and analysis of the output signal of the encoder gear speed using a mixer frequency measurement method: CD4070 XOR gate the two squarewave mixing, whereby the output frequency is input along with the frequency of the square wave with twice the speed, and square wave proportional relationship was CC2530 chip collection, and then to measure speed and distance measurement functions. Wherein the encoding tachometer circuit shown in Fig. (11). 

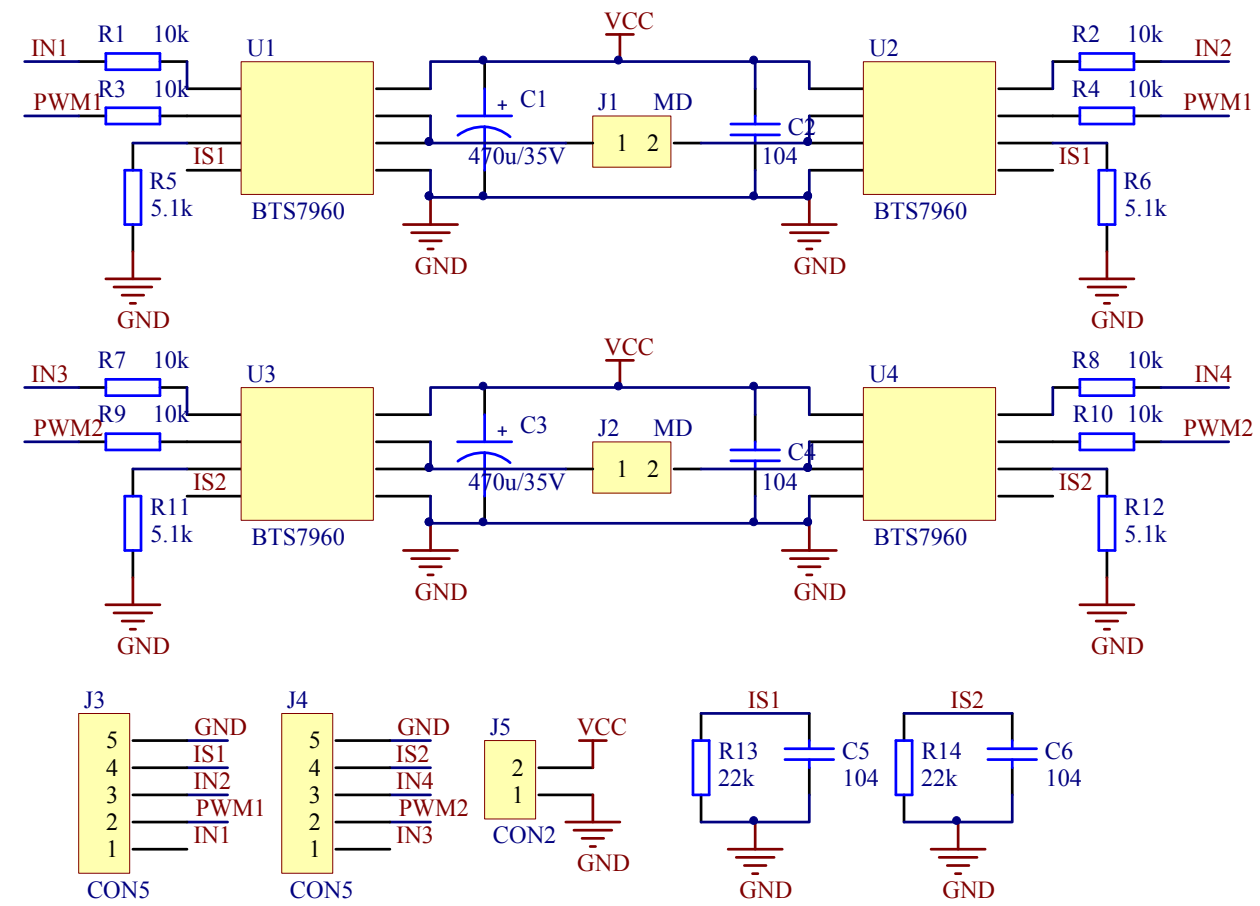

Fig. (10). BTS7960 dual H-bridge driver circuit.
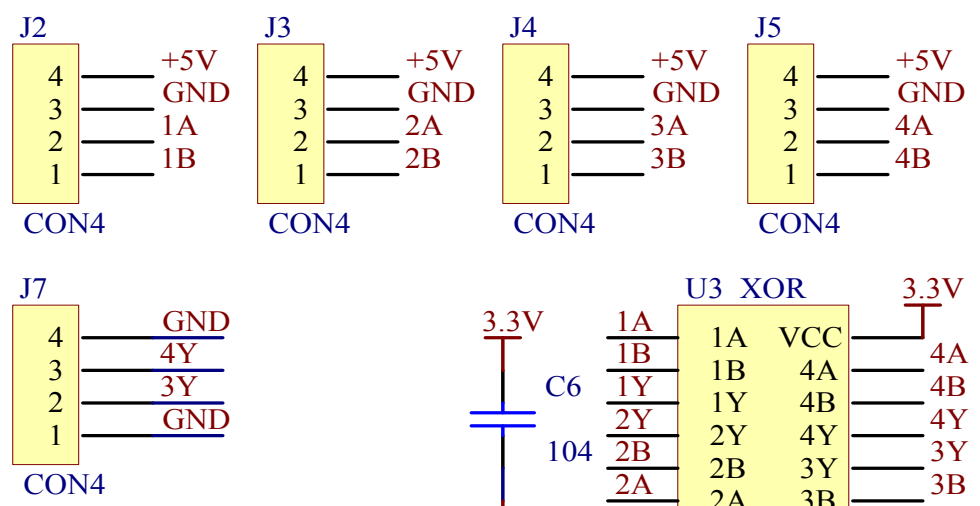

\section{Encoder}



Fig. (11). Encoding speed diagram.

\section{THE OVERALL DESIGN OF MULTI-ROBOT CONTROL}

Multi-robot control and overall management of the system mainly realized through the structure, the coordinator module device management and conservation of the network address of each robot. The router module and terminal equipment modules are planning to manage and store various types of sensor data and control parameters.

Wherein the coordinator module device CAR_MA$X \_N U M B$ defines the maximum number allowed in a network robot, the present value of the system 20. Meanwhile CAR structure of car_numb shows the current number of robots has joined the network, its value is less than or equal CAR_MAX_NUMB; size CAR_MAX_NUMB, type afAddrType_t stored in an array car_addr robot networks currently have joined the network address information to facilitate robot overall control and management; and finally an array of size CAR_MAX_NUMB car_function, storage arrays car_addr with corresponding functional status information indicates that the robot is mainly divided into fullfunction devices and reduced function devices, including 
streamlining the functions of the device can enter a lowpower sleep mode, the terminal equipment such as robots. The design of the code related definitions is as follows:

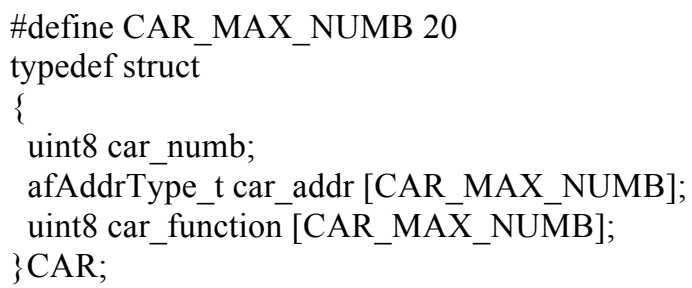

In addition, through the structure CAR_INFM achieve the router module and terminal equipment modules to manage and store various types of sensor data and control parameters:

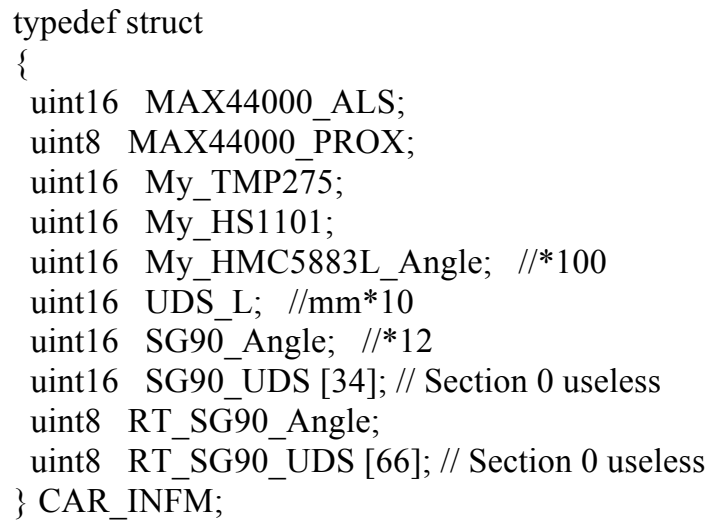

In ZigBee network multi-robot systems, the coordinator module, router modules and terminal equipment modules are based on the automatic start. After establishing the coordinator module ZigBee network, when the router module and terminal equipment modules discovered and join the network is complete, event messages through ZDO_STATE CHANGE application layer ZigBee network to transmit the network address assigned to the coordinator module, thus achieving the management and conservation of each a network address of the robot.

In addition, the design of various types of sensor data and control parameters management and overall control mainly by the router module and terminal equipment module function device implementation, while the PC is responsible for all types of robot data, aggregated parameters, analysis and related instructions the issued and so on. For example, the robot terminal apparatus A clock module is collected once every $1 \mathrm{~s}$ current temperature data of the temperature sensor TMP275, after treatment, to save the information to the robot A is My_TMP275 CAR_INFM structure; when a PC assigned to read the current environment in which the robot $\mathrm{A}$ when the temperature data, the robot a temperature data is extracted and sent to the ZigBee network coordinator, the coordinator module temperature data network label and robots a encapsulates processing, and then transmitted via the serial port to the $\mathrm{PC}$, the realization of the robot a temperature sensor data management. Overall control and management of the system of robot motion control and humidity sensors, ultrasonic sensors and other sensors and other modules are also using this method.

\section{CONCLUSION}

The system is based on wireless sensor network architecture based on the full use of PSoC5 and CC2530 resources, the use of wireless sensor networks, multi-robot technology and multi-sensor technology, to achieve the overall multirobot control functions. Through testing, the system has a stable, reliable communication, simple and diversified information collection characteristics, can be widely used in intelligent surveillance applications. At the same time, you can further use PSoC5 high performance CC2530 can be configured resources and network resources, according to the actual needs of extended-related functional modules, such as increasing the WiFi wireless module to complete the scene image wireless transmission function, can further improve the multi-robot system based PSoC5 and the CC2530 .

\section{CONFLICT OF INTEREST}

The author confirms that this article content has no conflicts of interest.

\section{ACKNOWLEDGEMENTS}

The authors are highly thankful for the Guangxi Natural Science Foundation (ID: 2013GXNSFBA019282), Research Program of science at Universities of Guang Xi Autonomous Region (ID: ZD2014112, 2013YB205), This research was financially supported by the project of outstanding young teachers' training in higher education institutions of Guangxi, Guangxi Colleges and Universities Key Laboratory Breeding Base of System Control and Information Processing.

\section{REFERENCES}

[1] J. Li, and Y. Hu, "Application design CC2530 ZigBee-based communications network," Electronic Design Engineering, vol. 16, pp. 108-111, 2011.

[2] J. Peng, X. Li, and Z. Qin,"Swarm-robot system based on wireless sensor network", Journal of Digital Information Management, vol. 10, no. 5, pp. 277-283, 2012.

[3] S. Tian, and X. Gao, "Design of ZigBee multi-robot communication system," Communication Technology, vol. 5, pp. 207-209, 2010.

[4] C Wang. Multi-Sensor Fusion Technology In Mobile Robot Application, North China University, Beijing, 2010

[5] B. Ma, "Research status and development of multi-robot systems," Science and Technology Information, vol. 4, pp. 504, 2009.

[6] X. Zhao, "On the technology and development of multi-robot," Equipment Manufacturing Technology, vol.5, pp. 141-142, 2009.

[7] G. Jing, and X. Deqian, "Research on key technologies of multiple mobile robots," Fujian computer, vol.1, pp. 5-6, 2009.

[8] Z. Bo, J. Wu, and M. Chen, "An intelligent robot system based on ZigBee technology," Audio-visual education research, vol. 9, no.11, pp.70-72,

[9] J. Peng, Q. He, and Q. Wei, "Design of smart things robot system", Information Technology Journal, vol. 12, no. 24, pp. 8514-8520, 2013. 
[10] P. Na, Reticular, ZigBee Wireless Sensor Networks, Jilin University, Jilin, 2010.

[11] A True System-on-Chip Solution for 2.4-GHz IEEE 802.15.4 and ZigBee Applications. Texas Instruments [DB/OL]. Available at: http://www.ti.com/lit/ds/swrs081b/swrs081b.pdf, 2012.

[12] PSoC(R) Creator(TM) System Reference Guide V2.40. Cypress Semiconductor [DB/OL] Available at: http://www.cypress.com $/$ ?docID=32460, 2011 .
[13] WKA, MKEA.PSoC®5:CY8C55 Family Datasheet Programmable System-on-Chip. Cypress Semiconductor [DB/OL]. http://www.cypress.com/, 2011.

[14] C. Jun, and Y. Li "HS1101 humidity sensor in the smart home control system application", Electronic Testing, vol. 2, pp. 77-80, 2008.

Received: September 16, 2014

(C) Jiansheng Peng; Licensee Bentham Open.

This is an open access article licensed under the terms of the Creative Commons Attribution Non-Commercial License (http://creativecommons.org/licenses/by-nc/3.0/) which permits unrestricted, non-commercial use, distribution and reproduction in any medium, provided the work is properly cited. 